\title{
Students' perception of the educational environment of medical schools in Korea: findings from a nationwide survey
}

Kwi Hwa Park', Joo Hyun Park², Sun Kim³ , Jung Ae Rhee ${ }^{4}$, Jong Hoon Kim, Young Joon Ahn ${ }^{6}$, Jae Jin $\mathrm{Han}^{7}$ and Duk Joon $\mathrm{Suh}^{8}$

${ }^{1}$ Department of Medical Education, Gachon University College of Medicine, Incheon, ${ }^{2}$ Department of Medical Education, University of Ulsan College of Medicine, ${ }^{3}$ Department of Medical Education, The Catholic University of Korea College of Medicine, Seoul, ${ }^{4}$ Department of Preventive Medicine, Chonnam National University Medical School, Gwangju, ${ }^{5}$ Department of Medical Education, Inha University College of Medicine, Incheon, ${ }^{6}$ Department of Medical Education, Chosun University College of Medicine, Gwangju, ${ }^{7}$ Department of Medical Education, Ewha Womans University College of Medicine, Seoul, and ${ }^{8}$ Department of Physiology, Dong-A University College of Medicine, Busan, Korea

\section{한국 의과대학생들의 의학교육환경에 대한 인식}

${ }^{1}$ 가천대학교 의과대학 의학교육학과, ${ }^{2}$ 울산대학교 의과대학 의학교육학교실, ${ }^{3}$ 가톨릭대학교 의과대학 의학교육학과, ${ }^{4}$ 전남대학교 의과대학 예방의학교실, ${ }^{5}$ 인하대학교 의과대학 의학교육학교실, ${ }^{6}$ 조선대학교 의과대학 의학교육학교실, ${ }^{7}$ 이화여자대학교 의과대학 의학교육학교실, ${ }^{8}$ 동아대학교 의과대학 생리학교실

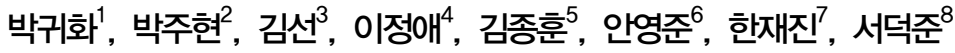

Purpose: The purpose of this study was to examine students' perception of the educational environment of medical schools in Korea. Methods: A total of 9,096 of 12,035 students (75.6\%) responded to our questionnaire. This study was conducted at the end of the 2013 academic year using the Dundee Ready Education Environment Measure (DREEM) at 40 medical schools in Korea. DREEM comprises five domains: students' perceptions of learning (SPL); students' perceptions of teachers (SPT); students' academic self-perceptions; students' perceptions of atmosphere; and students' social self-perception. The data were analyzed using descriptive statistics, independent t-test, and one-way analysis of variance.

Results: The overall mean DREEM score was 113.97 (of 200), and the scores for the 40 medical schools ranged from 100.24 to 134.32. The overall mean and domains scores of the DREEM differed significantly between educational systems, grades, genders, and academic achievement levels. Graduate-level medical students had higher scores for the DREEM and its five domains than undergraduate medical students. The scores were lowest in second-year students (mean, 111.80). Male students' perceptions were significantly higher than those of female students except for SPL and SPT. High academic achievers' perceptions were also greater versus low academic achievers.

Conclusion: Students' perceptions of their educational environment are positive in Korea. The learning environment should be evaluated by curriculum planners and administrators of medical schools to improve its quality.

Key Words: Dundee Ready Education Environment Measure, Learning environment, Medical education, Medical students

Received: February 26, 2015 • Revised: March 29, 2015 • Accepted: March 29, 2015 Corresponding Author: Joo Hyun Park (http://orcid.org/0000-0001-8029-0316) Department of Medical Education, University of Ulsan College of Medicine, 88 Olympic-ro 43 gil, Songpa-gu, Seoul 138-736, Korea

Tel: +82.2.3010.4268 Fax: +82.2.3010.8465 email: doogie@amc.seoul.kr
Korean J Med Educ 2015 Jun; 27(2): 117-130 http://dx.doi.org/10.3946/kjme.2015.27.2.117 eISSN: $2005-7288$

(C) The Korean Society of Medical Education. All rights reserved. This is an open-access article distributed under the terms of the Creative Commons Attribution Non-Commercial License (http:// creativecommons.org/licenses/by-nc/3.0/), which permits unrestricted non-commercial use, distribution, and reproduction in any medium, provided the original work is properly cited. 


\section{서론}

학생들의 학습에 영향을 미치는 요인은 매우 다양하다. 그 중에서도 교육과정, 교수법, 지원체계, 물리적 환경과 같은 교 육환경이 중요한 요인 중 하나이다[1]. 학생들이 어떤 교육환 경에 처해 있고, 그 환경을 어떻게 인식하고 있는지를 파악하 여 개선하는 것은 학습을 촉진시키고, 학업성취도를 향상시 키는 데 도움이 된다[2,3]. 따라서 효율적으로 학습할 수 있는 적절한 교육환경을 제공하는 것이 중요하다.

교육환경은 학습자가 대학의 교육과정 이수를 위해 상호작 용하는 모든 물리적, 심리적, 사회적 맥락을 의미한다[4]. 교 육환경을 평가하는 것은 질 높은 의학교육과정을 제공하기 위한 핵심 활동이며, 교육환경에 대한 학생들의 피드백은 교 육환경 개선을 위한 가장 중요한 근거자료가 된다[2]. 따라서 학생들이 의학교육환경에 대해 어떻게 인식하고 있는지를 평 가하기 위해서는 신뢰할 만하고 타당한 도구를 사용하는 것 이 중요하다.

지난 17 년 동안, 의학뿐만 아니라 의료 관련 분야에서 교육 환경의 강점과 약점을 피드백하고 개선하기 위해 가장 보편 적으로 사용되어 온 도구가 Dundee Ready Education Environment Measure (DREEM)이다[5]. DREEM은 간호 학, 치의학 등과 같은 전공분야에서도 적용하고 있지만, 의과 대학을 대상으로 한 연구에 집중적으로 사용되어 왔다[6]. 영 국에서 개발하였지만, 전 세계 20개국에서 사용하고 있고, 8 개 이상의 언어로 번역되어 널리 사용되고 있다[7].

DREEM은 학습(students' perception of learning, SPL), 교수(students' perceptions of teachers, SPT), 학업(students' academic self-perceptions, SAS), 분위기(students' perceptions of atmosphere, SPA), 사회적 관계(students' social self-perceptions, SSS)의 5 개 하위 요인으로 구성되 어 있으며, 이러한 교육환경 요인에 대해 어떻게 인식하고 있 는지를 평가하는 도구이다[5]. 지금까지 DREEM은 의학교육 과정 개편 전후에 변화를 평가하거나[8], 개별 의과대학의 의 학교육환경에 대한 강점과 약점을 파악하거나[9], 다른 의과 대학의 교육환경과 비교하거나[10], 학생들의 성취와의 관련 성을 연구[11]하는 등에 다양하게 활용되어 왔다. 이처럼 외
국에서는 의학교육환경에 대해 관심을 갖고 활발한 연구를 진행하고 있는 것에 비해 국내 연구는 드물다.

지금까지 우리나라 의학교육은 괄목할 만한 변화와 발전을 해 왔고[12], 외국의 다양한 제도와 교육과정을 도입하려는 노력을 해 왔다. 이제는 국내에서도 의학교육환경에 관심을 갖고 조사해 볼 때이다. 학생들이 지금의 우리나라 의학교육 환경에 대해 어떻게 인식하고 있으며, 다른 나라와 비교하여 어느 정도 수준이며, 어떠한 차이가 있는지를 객관적으로 조 사해 볼 필요가 있다.

따라서 본 연구에서는 우리나라 의과대학 (이하 의대) 및 의학전문대학원(이하 의전원)을 대상으로 전수조사를 실시하 여 학생들이 의학교육환경에 대해 어떻게 인식하고 있는지를 파악하고, 향후 의학교육환경의 중요성을 인식시키고 개선방 향을 모색하는 데 기초자료를 제공하고자 한다.

\section{대상 및 방법}

\section{1. 연구 대상}

본 연구는 전국 41 개 의대 또는 의전원에 재학 중인 1학년 에서 4학년 학생 전체를 대상으로 전수조사를 실시하였다. 41 개 대학 중 1 개 대학을 제외한 40 개 대학이 회신을 하였다. 각 대학의 입학정원을 기준으로 총 학생 수는 약 12,035 명이

\begin{tabular}{lcc}
\hline Table 1. Demographics of Subjects \\
\hline \multicolumn{2}{c}{ Variable } & No. \\
\hline Educational system & $\%$ \\
UMS & 3,892 & 43.1 \\
GEMS & 5,134 & 56.9 \\
Gender & & \\
Male & 5,317 & 60.7 \\
Female & 3,439 & 39.3 \\
Grade & & \\
1st year & 2,460 & 27.3 \\
2nd year & 2,279 & 25.3 \\
3rd year & 2,409 & 26.7 \\
4th year & 1,862 & 20.7 \\
\hline
\end{tabular}

UMS: Undergraduate medical student, GEMS: Graduate entry medical student. 
며, 이 중 9,096부가 회수되어(회수율 약 $75.6 \%$ ), 이를 최종 분석 대상으로 하였다.

연구 대상의 분포를 살펴보면, 의대생이 3,892명(43.1\%), 의 전원생이 5,134명(56.9\%)이며, 남학생이 5,317명(60.7\%), 여 학생이 3,439명(39.3\%)이었다. 학년 분포는 1학년이 2,460명 (27.3\%), 2학년이 2,279명(25.3\%), 3학년이 2,409명(26.7\%), 4 학년이 1,862명(20.7\%)이었다(Table 1). 평균 연령은 25.54 세(표준편차 3.34)였으며, 연령범위는 19세에서 58세였다.

\section{2. 연구 도구}

\section{1) 교육환경인식 척도}

학생들이 의학교육환경을 어떻게 인식하는지를 측정하기 위 해 Roff et al. [5]이 개발한 DREEM을 번안하여 사용하였다. 의학교육환경은 학생이 지각하는 학교 및 강의실의 분위기, 학 업에 대한 확신 또는 자신감, 학교에서의 사회적 관계, 학생이 학습 또는 수업, 교수에 대해 갖고 있는 태도와 인식 등을 말한 다[5]. DREEM은 5개 하위요인으로 구성되어 있으며, 각 요인 별 문항 수는 SPL 12문항, SPT 11문항, SAS 8문항, SPA 12문 항, SSS 7문항으로 총 50문항이다(Appendix 1).

DREEM의 총점은 200점이며, 총점에 대한 해석은 very poor (0 50점), plenty of problem (51 100점), more positive than negative (101 150점), excellent (151 200점)을 기준으로 한다. 각 문항에 대한 해석은 positive (3.5 이상), could be enhanced or improved (2 3점), problematic area (2점 이하)를 기준으로 한다[13].

각 문항에 대한 반응양식은 리커트(Likert) 5점 척도이며, '전혀 그렇지 않다'의 0 점부터 '매우 그렇다의 4점까지 반응하 도록 하였다. 역채점 문항은 9 개 $(4,8,9,17,25,35,39,48$, 50) 문항이다. 각 하위 요인별 점수가 높을수록 해당 요인에 대해 긍정적이고 만족스럽게 인식하고 있는 것으로 해석한다. 본 연구에서 하위 요인별 Cronbach $\alpha$ 계수는 0.66 에서 0.85 의 범위(SPL, 0.85; SPT, 0.78; SAS, 0.77; SPA, 0.79; SSS, 0.66)였고, 전체 Cronbach $\alpha$ 계수는 0.94 였다.

\section{2) 학업성적}

학생들의 학업성적은 학생이 스스로 지각한 학업성적 수준 에 대한 반응으로 자기보고식 평가로 알아보았다. 직전 학기 의 학업성적에 대해 상위권, 중상위권, 중위권, 중하위권, 하
위권으로 구분하여 응답하도록 하였다. 결과 분석 시에는 상 위권과 중상위권은 상위권으로, 중하위권과 하위권은 하위권 으로 재분류하여 분석하였다.

\section{3. 자료 수집}

본 연구의 자료 수집을 위해 한국의학교육학회 주관으로 2013년 11월부터 2014년 3월까지 전국 의대 또는 의전원 학 생을 대상으로 설문조사를 실시하였다. 각 의대 학장 또는 의 전원 원장에게 연구 목적과 설문 시 주의사항 등이 포함된 공 문을 발송하여 협조를 요청하였다. 설문지 회수는 설문이 완 료된 후에 연구자에게 회송하는 방식으로 진행되었다. 설문 지 작성은 학년별 집단으로 시행하였으며, 학생들이 설문지 를 완성하는 데 소요되는 시간은 약 15 분이었다. 분석이 종료 된 후에 각 대학에 분석 결과를 송부하여 대학에서 활용하도 록 하였다.

\section{4. 결과 분석}

본 연구에서 사용된 결과 분석 방법은 다음과 같다. 첫째, DREEM의 점수분포를 알아보기 위해 평균과 표준편차를 구 하였다. 둘째, 학생의 학제와 성별에 따른 DREEM의 차이를 살펴보기 위해 각각 독립표본 $\mathrm{t}$-검정을 실시하였다. 셋째, 학 생의 학년과 학업성적 수준에 따른 DREEM의 차이를 살펴보 기 위해 각각 one-way analysis of variance를 실시하였다.

\section{결과}

\section{DREEM의 평균점수 및 대학별 평균점수 분포}

\section{1) DREEM의 평균점수 분포}

$\mathrm{DREEM}$ 의 전체 평균점수와 하위 요인별 평균점수를 분석 하였다. 그 결과 우리나라 DREEM 전체 평균점수는 200점 만점 중 113.97점이며, 각 하위 요인별 평균점수는 SPL이 26.19점, $\mathrm{SPT}$ 가 26.57점, SAS가 18.81점, SPA가 26.54점, $\mathrm{SSS}$ 가 15.76점이었다. 또한 DREEM 전체 평균점수와 각 하 위 요인별 평균점수를 100점 만점으로 환산하면, 우리나라 DREEM 전체 평균점수는 56.99점이며, 하위 요인인 SPL은 
54.57점, SPT는 60.40점, SAS는 58.79점, SPA가 55.29점, $\mathrm{SSS}$ 가 56.28점이었다. 즉, $\mathrm{SPT}$ 에서 가장 높은 점수를 나타내 었고, SPL에서 가장 낮은 점수를 나타내었다(Table 2).

2) 대학별 DREEM의 평균점수 분포

연구 대상인 40 개 대학의 대학별 DREEM 전체 평균점수는
최고 134.32점에서 최하 100.24점까지 분포하였다. 전체 평 균점수가 130점 이상인 대학은 1개 대학, 130점에서 120점까 지인 대학은 10 개 대학, 120 점에서 110 점까지인 대학은 17 개 대학, 110점 미만인 대학은 12 개 대학이었다(Table 3).

Table 2. Mean Total and Subscale DREEM Scores

\begin{tabular}{cccccc}
\hline Domain of DREEM & No. & Maximum score & Mean & SD & $\%$ \\
\hline SPL & 8,804 & 48 & 26.19 & 6.31 & 54.57 \\
SPT & 8,684 & 44 & 26.57 & 4.98 & 60.40 \\
SAS & 8,829 & 32 & 18.81 & 4.24 & 58.79 \\
SPA & 8,485 & 48 & 26.54 & 5.99 & 55.29 \\
SSS & 8,833 & 28 & 15.76 & 3.70 & 56.28 \\
Total & 8,097 & 200 & 113.97 & 21.59 & 56.99 \\
\hline
\end{tabular}

DREEM: Dundee Ready Education Environment Measure, SD: Standard deviation, \%: Conversion to 100-point scale, SPL: Students' perceptions of learning, SPT: Students' perceptions of teachers, SAS: Students' academic self-perceptions, SPA: Students' perceptions of atmosphere, SSS: Students' social self-perceptions.

Table 3. Mean Total and Subscale DREEM Scores in 40 Medical Schools

\begin{tabular}{|c|c|c|c|c|c|c|c|}
\hline Medical school & SPL & SPT & SAS & SPA & SSS & Total & $\%$ \\
\hline 1 & 31.52 & 31.06 & 20.96 & 32.24 & 18.24 & 134.32 & 67.16 \\
\hline 2 & 29.66 & 28.97 & 20.12 & 29.53 & 17.51 & 125.81 & 62.90 \\
\hline 3 & 28.38 & 29.72 & 19.77 & 28.59 & 17.13 & 123.56 & 61.78 \\
\hline 4 & 28.31 & 29.22 & 19.57 & 28.72 & 17.28 & 122.76 & 61.38 \\
\hline 5 & 28.31 & 28.72 & 19.34 & 28.63 & 16.46 & 122.14 & 61.07 \\
\hline 6 & 27.22 & 28.32 & 19.78 & 28.48 & 16.67 & 121.06 & 60.53 \\
\hline 7 & 27.71 & 27.65 & 19.85 & 28.31 & 17.52 & 120.90 & 60.45 \\
\hline 8 & 27.83 & 28.23 & 19.67 & 28.57 & 16.85 & 120.79 & 60.39 \\
\hline 9 & 28.65 & 27.65 & 19.59 & 27.59 & 17.01 & 120.56 & 60.28 \\
\hline 10 & 28.68 & 27.97 & 19.30 & 28.29 & 16.64 & 120.49 & 60.25 \\
\hline 11 & 27.67 & 28.59 & 19.35 & 28.29 & 16.41 & 120.38 & 60.19 \\
\hline 12 & 28.29 & 27.03 & 19.27 & 26.92 & 16.53 & 117.65 & 58.83 \\
\hline 13 & 26.99 & 26.43 & 20.11 & 26.98 & 17.04 & 117.64 & 58.82 \\
\hline 14 & 26.30 & 27.01 & 19.53 & 27.42 & 17.47 & 116.92 & 58.46 \\
\hline 15 & 27.14 & 28.82 & 18.31 & 26.46 & 14.83 & 116.41 & 58.21 \\
\hline 16 & 26.87 & 27.17 & 19.26 & 26.15 & 16.47 & 116.34 & 58.17 \\
\hline 17 & 27.35 & 27.74 & 19.29 & 27.66 & 15.06 & 116.01 & 58.00 \\
\hline 18 & 26.70 & 27.34 & 18.54 & 27.26 & 16.18 & 115.82 & 57.91 \\
\hline 19 & 25.66 & 27.10 & 18.99 & 26.29 & 15.66 & 113.88 & 56.94 \\
\hline 20 & 26.51 & 26.30 & 18.48 & 26.49 & 15.42 & 113.47 & 56.74 \\
\hline 21 & 26.81 & 25.94 & 19.01 & 25.82 & 15.30 & 113.26 & 56.63 \\
\hline 22 & 25.46 & 26.60 & 18.42 & 27.11 & 14.79 & 112.76 & 56.38 \\
\hline 23 & 26.72 & 26.27 & 18.34 & 26.09 & 15.28 & 112.71 & 56.36 \\
\hline 24 & 25.97 & 26.33 & 18.45 & 26.02 & 15.26 & 111.97 & 55.99 \\
\hline 25 & 25.65 & 26.07 & 18.24 & 26.36 & 15.75 & 111.92 & 55.96 \\
\hline 26 & 26.17 & 25.70 & 18.73 & 25.67 & 15.50 & 111.91 & 55.95 \\
\hline
\end{tabular}

(Continued to the next page) 
Table 3. (Continued)

\begin{tabular}{cccccccc}
\hline Medical school & SPL & SPT & SAS & SPA & SSS & Total & $\%$ \\
\hline 27 & 25.49 & 26.23 & 18.31 & 26.55 & 14.67 & 111.60 & 55.80 \\
28 & 25.44 & 25.86 & 19.01 & 26.29 & 15.13 & 111.40 & 55.70 \\
29 & 25.53 & 26.19 & 18.45 & 25.62 & 14.52 & 109.93 & 54.96 \\
30 & 24.35 & 25.34 & 18.49 & 25.66 & 15.46 & 108.95 & 54.48 \\
31 & 23.73 & 25.19 & 17.86 & 25.42 & 15.46 & 108.34 & 54.17 \\
32 & 24.67 & 24.56 & 19.20 & 24.97 & 14.77 & 108.26 & 54.13 \\
33 & 24.77 & 24.19 & 18.24 & 24.93 & 14.86 & 106.99 & 53.50 \\
34 & 23.81 & 25.80 & 18.21 & 25.14 & 14.63 & 106.49 & 53.25 \\
35 & 23.69 & 25.36 & 17.64 & 25.20 & 14.37 & 106.31 & 53.15 \\
36 & 23.29 & 24.68 & 18.17 & 24.23 & 14.76 & 106.17 & 53.09 \\
37 & 24.11 & 24.93 & 17.15 & 24.16 & 14.72 & 104.95 & 52.48 \\
38 & 23.84 & 24.71 & 17.66 & 24.23 & 14.09 & 104.41 & 52.21 \\
39 & 21.98 & 24.49 & 17.30 & 24.71 & 13.30 & 102.09 & 51.05 \\
40 & 22.53 & 24.76 & 16.52 & 22.88 & 13.63 & 100.24 & 50.12 \\
Mean & 26.19 & 26.57 & 18.81 & 26.54 & 15.76 & 113.97 & 56.99 \\
\hline
\end{tabular}

DREEM: Dundee Ready Education Environment Measure, \%: Conversion to 100-point scale, SPL: Students' perceptions of learning, SPT: Students' perceptions of teachers, SAS: Students' academic self-perceptions, SPA: Students' perceptions of atmosphere, SSS: Students' social self-perceptions.

\begin{tabular}{|c|c|c|c|}
\hline Item & No. & Mean & SD \\
\hline \multicolumn{4}{|l|}{ SPL } \\
\hline 1. I am encouraged to participate in class & 8,980 & 2.68 & 0.84 \\
\hline 7. The teaching is often stimulating & 8,980 & 2.21 & 0.86 \\
\hline 13. The teaching is student-centered & 8,986 & 1.90 & 0.91 \\
\hline 16. The teaching helps to develop my competence & 8,972 & 2.58 & 0.78 \\
\hline 20. The teaching is well focused & 8,970 & 2.45 & 0.75 \\
\hline 22. The teaching helps to develop my confidence & 8,976 & 2.12 & 0.88 \\
\hline 24. The teaching time is put to good use & 8,967 & 2.32 & 0.83 \\
\hline 25. The teaching over-emphasizes factual learning ${ }^{\text {al }}$ & 8,974 & 1.81 & 0.92 \\
\hline 38. I am clear about the learning objectives of the course & 8,959 & 2.26 & 0.74 \\
\hline 44. The teaching encourages me to be an active learner & 8,967 & 2.04 & 0.88 \\
\hline 47. Long-term learning is emphasized over short-term learning & 8,956 & 1.92 & 0.99 \\
\hline 48. The teaching is too teacher-centered ${ }^{\mathrm{al}}$ & 8,957 & 1.90 & 0.83 \\
\hline \multicolumn{4}{|l|}{ SPT } \\
\hline 2. The teachers are knowledgeable & 8,992 & 3.27 & 0.69 \\
\hline 6. The teachers are patient with patients & 8,928 & 2.57 & 0.73 \\
\hline 8. The teachers ridicule the students ${ }^{\mathrm{al}}$ & 8,982 & 2.41 & 0.90 \\
\hline 9. The teachers are authoritarian ${ }^{\mathrm{a})}$ & 8,985 & 1.93 & 0.94 \\
\hline 18. The teachers have good communications skills with patients & 8,800 & 2.51 & 0.73 \\
\hline 29. The teachers are good at providing feedback to students & 8,976 & 2.10 & 0.86 \\
\hline 32. The teachers provide constructive criticism here & 8,972 & 2.48 & 0.78 \\
\hline 37. The teachers give clear examples & 8,971 & 2.46 & 0.73 \\
\hline 39. The teachers get angry in class a & 8,963 & 2.00 & 0.98 \\
\hline 40. The teachers are well prepared for their classes & 8,959 & 2.60 & 0.76 \\
\hline 50. The students irritate the teachers ${ }^{\text {al }}$ & 8,962 & 2.26 & 0.83 \\
\hline
\end{tabular}

(Continued to the next page) 
Table 4. (Continued)

\begin{tabular}{|c|c|c|c|}
\hline Item & No. & Mean & SD \\
\hline \multicolumn{4}{|l|}{ SAS } \\
\hline 5. Learning strategies which worked for me before continue to work for me now & 8,993 & 1.80 & 1.05 \\
\hline 10. I am confident about my passing this year & 8,981 & 2.98 & 0.88 \\
\hline 21. I feel I am being well prepared for my profession & 8,979 & 2.33 & 0.80 \\
\hline 26. Last year's work has been a good preparation for this year's work & 8,929 & 2.42 & 0.87 \\
\hline 27. I am able to memorize all I need & 8,967 & 1.41 & 0.92 \\
\hline 31. I have learned a lot about empathy in my profession & 8,969 & 2.62 & 0.73 \\
\hline 41. My problem-solving skills are being well developed here & 8,957 & 2.42 & 0.78 \\
\hline 45. Much of what I have to learn seems relevant to a career in healthcare & 8,958 & 2.80 & 0.77 \\
\hline \multicolumn{4}{|l|}{ SPA } \\
\hline 11. The atmosphere is relaxed during the ward teaching & 8,626 & 2.03 & 0.79 \\
\hline 12. This school is well time-tabled & 8,974 & 2.24 & 0.96 \\
\hline 17. Cheating is a problem in this school $\left.\right|^{a}$ & 8,972 & 2.57 & 1.21 \\
\hline 23. The atmosphere is relaxed during lectures & 8,975 & 2.39 & 0.80 \\
\hline 30. There are opportunities for me to develop interpersonal skills & 8,976 & 2.19 & 0.97 \\
\hline 33. I feel comfortable in class socially & 8,978 & 2.45 & 0.83 \\
\hline 34. The atmosphere is relaxed during seminars/tutorials & 8,945 & 2.50 & 0.79 \\
\hline 35. I find the experience disappointing ${ }^{\text {al }}$ & 8,965 & 1.62 & 0.91 \\
\hline 36. I am able to concentrate well & 8,961 & 2.33 & 0.76 \\
\hline 42. The enjoyment outweighs the stress of the course & 8,961 & 1.96 & 0.96 \\
\hline 43. The atmosphere motivates me as a learner & 8,958 & 2.19 & 0.85 \\
\hline 49. I feel able to ask the questions I want & 8,960 & 2.06 & 0.99 \\
\hline \multicolumn{4}{|l|}{ SSS } \\
\hline 3. There is a good support system for students who get stressed & 8,984 & 1.43 & 0.96 \\
\hline 4. I am too tired to enjoy the course $\mathrm{a}^{\mathrm{a}}$ & 8,951 & 2.15 & 0.93 \\
\hline 14. I am rarely bored on this course & 8,969 & 2.24 & 1.00 \\
\hline 15. I have good friends in this school & 8,976 & 3.05 & 0.76 \\
\hline 19. My social life is good & 8,972 & 2.86 & 0.71 \\
\hline 28. I seldom feel lonely & 8,972 & 2.01 & 0.97 \\
\hline 46. My accommodation is pleasant & 8,959 & 2.02 & 1.08 \\
\hline
\end{tabular}

DREEM: Dundee Ready Education Environment Measure, SD: Standard deviation, SPL: Students' perceptions of learning, SPT: Students' perceptions of teachers, SAS: Students' academic self-perceptions, SPA: Students' perceptions of atmosphere, SSS: Students' social self-perceptions.

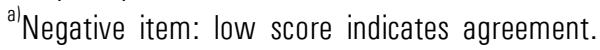

\section{DREEM의 문항별 평균점수}

DREEM의 문항별 점수는 평균점수 2점 이하와 평균점수 3점 이상인 문항으로 구분하여 분석하였다(Table 4). 평균점수가 2점 이하로 낮았던 문항을 하위 요인별로 살펴 보면, SPL 요인에서는 "장기기억의 학습이 단기기억의 학습 보다 강조된다(mean \pm standard deviation, $1.92 \pm 0.99)$ ", “수 업은 학생 중심적이다(1.90 \pm 0.91$)$ ", “수업이 너무 교수 중심 적이다( $1.90 \pm 0.83)$ ", “수업은 사실 자체의 단순암기학습(단
순지식전달)을 지나치게 강조한다( $1.81 \pm 0.92) ”$ 의 4문항이다.

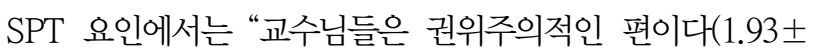
0.94)"의 1문항이다. SAS 요인에서는 “과거에 내가 공부할 때 사용했던 학습 전략(방법)이 지금 의대 공부를 하는 데도 효

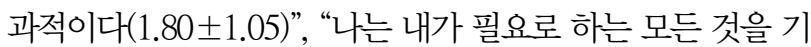
억할 수 있다( $1.41 \pm 0.92) "$ 의 2문항이며, 27번 문항은 50개 문항 중에서도 가장 낮은 평균점수를 나타내었다. SPA 요인 에서는 "나는 학업에 대해 스트레스를 받기보다는 즐기는 편 이다(1.96 \pm 0.96$)$ ", "나는 의대/의전원에서 실망스러운 경험 
Table 5. Students' Perception of DREEM by Educational System

\begin{tabular}{ccccccc}
\hline Factor & Educational system & No. & Mean & SD & t & p-value \\
\hline SPL & UMS & 3,780 & 25.19 & 6.41 & -13.168 & 0.000 \\
& GEMS & 5,019 & 26.96 & 6.12 & & \\
SPT & UMS & 3,733 & 26.00 & 4.80 & -9.366 & 0.000 \\
& GEMS & 4,947 & 27.01 & 5.06 & & \\
SAS & UMS & 3,815 & 18.51 & 4.47 & -5.951 & 0.000 \\
& GEMS & 5,009 & 19.05 & 4.03 & & \\
SPA & UMS & 3,659 & 25.82 & 6.02 & -9.670 & 0.000 \\
& GEMS & 4,822 & 27.09 & 5.90 & & \\
SSS & UMS & 3,797 & 15.44 & 3.75 & -7.169 & 0.000 \\
\multirow{2}{*}{ Total } & GEMS & 5,031 & 16.00 & 3.64 & & \\
& UMS & 3,494 & 111.02 & 21.64 & -10.827 & 0.000 \\
& GEMS & 4,599 & 116.23 & 21.28 & & \\
\hline
\end{tabular}

DREEM: Dundee Ready Education Environment Measure, SD: Standard deviation, SPL: Students' perceptions of learning, UMS: Undergraduate medical student, GEMS: Graduate entry medical student, SPT: Students' perceptions of teachers, SAS: Students' academic self-perceptions, SPA: Students' perceptions of atmosphere, SSS: Students' social self-perceptions.

을 하곤 한다(1.62 \pm 0.91$)$ "의 2문항이다. SSS 요인에서는 "스

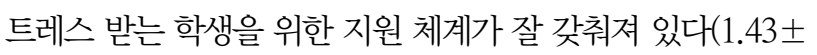
0.96)"의 1 문항이다.

평균점수가 3점 이상으로 높았던 문항을 하위 요인별로 살 펴보면, SPT 요인의 “교수님들은 풍부한 지식을 갖고 있다 (3.27 \pm 0.69$)$ " 문항과 SSS 요인의 "나는 학교에 친한 친구들 이 있다( $3.05 \pm 0.76)$ " 문항이다. 이 중 2 번 문항은 50 개 문항 중 가장 높은 평균점수를 나타내었다.

\section{3. 학제에 따른 DREEM의 차이}

의대생과 의전원생 두 집단의 DREEM의 차이를 분석한 결 과, DREEM 전체 평균점수는 의전원생이 116.23 점이고 의대 생이 111.02점으로 나타나, 의전원이 의대생보다 높았다 $(\mathrm{t}=$ $-10.827, \mathrm{p}<0.001)$. 각 하위 요인별로 살펴보면, $\mathrm{SPL}(\mathrm{t}=$ $-13.168, \mathrm{p}<0.001)$, SPT $(\mathrm{t}=-9.366, \mathrm{p}<0.001)$, SAS $(\mathrm{t}=$ $-5.951, \mathrm{p}<0.001)$, SPA $(\mathrm{t}=-9.670, \mathrm{p}<0.001)$, SSS $(\mathrm{t}=$ -7.169, $\mathrm{p}<0.001)$ 에서도 모두 의전원생이 의대생보다 높게 나타났다(Table 5). 즉, 의전원생이 의대생보다 의학교육환경 에 대해 더 긍정적으로 인식하는 것으로 나타났다.

\section{4. 학년에 따른 DREEM의 차이}

학년에 따른 DREEM의 차이를 분석한 결과, DREEM 전체
평균점수는 1학년 114.10점, 2학년 111.80점, 3학년 115.56점, 4학년 114.35점으로 나타나, 3학년이 가장 높았고, 2학년이 가 장 낮았다 $(\mathrm{F}=10.996, \mathrm{p}<0.001)$. 각 하위 요인별로 살펴보면, SPL $(F=19.533, p<0.001)$, SPT $(F=19.706, p<0.001)$, SAS $(\mathrm{F}=50.508, \quad \mathrm{p}<0.001), \quad \mathrm{SPA} \quad(\mathrm{F}=8.025, \quad \mathrm{p}<0.001), \quad$ SSS $(\mathrm{F}=42.726, \mathrm{p}<0.001)$ 에서도 모두 학년 간 유의미한 차이가 있 었다. SPL 요인은 2학년이 다른 학년에 비해 유의미하게 낮은 점수를 나타내었다. SPT 요인은 1 학년이 가장 높은 점수를 나 타내었으며, 학년이 높아질수록 유의미하게 낮은 점수를 나타 내었다. SAS 요인은 4학년이 가장 높은 점수를 나타내었으며, 3,4 학년이 1, 2학년보다 유의미하게 높은 점수를 나타내었다. $\mathrm{SPA}$ 요인은 1학년이 다른 학년에 비해 유의미하게 높은 점수 를 나타내었다. SSS 요인은 4학년이 가장 높은 점수를 나타내 었으며, 3, 4학년이 1, 2학년보다 유의미하게 높은 점수를 나타 내었다(Table 6).

\section{5. 성별에 따른 DREEM의 차이}

남학생과 여학생으로 구분하여 DREEM의 차이를 분석한 결과, DREEM 전체 평균점수는 남학생이 114.59점이고 여학 생이 113.01점으로 나타나, 남학생이 여학생보다 높았다 $(\mathrm{t}=$ $3.146, \mathrm{p}<0.01)$. 각 하위 요인별로 살펴보면, $\mathrm{SAS}(\mathrm{t}=3.668$, $\mathrm{p}<0.001)$, SPA $(\mathrm{t}=6.849, \mathrm{p}<0.001)$, SSS $(\mathrm{t}=5.644, \mathrm{p}<$ 
Table 6. Students' Perception of DREEM by Year

\begin{tabular}{|c|c|c|c|c|c|c|c|}
\hline Factor & Grade & No. & Mean & SD & $F$ & $p$-value & Scheffé \\
\hline \multirow[t]{4}{*}{ SPL } & $1^{\mathrm{al}}$ & 2,399 & 26.12 & 6.79 & 19.533 & 0.000 & $a>b, c>a, c>b, d>b$ \\
\hline & $2^{b /}$ & 2,229 & 25.44 & 6.20 & & & \\
\hline & $3^{c 1}$ & 2,343 & 26.84 & 6.03 & & & \\
\hline & $4^{d l}$ & 1,814 & 26.39 & 6.05 & & & \\
\hline \multirow[t]{4}{*}{ SPT } & $1^{\text {al }}$ & 2,302 & 27.11 & 5.36 & 19.706 & 0.000 & $a>b, a>c, a>d, b>d, c>d$ \\
\hline & $2^{\text {b) }}$ & 2,186 & 26.61 & 4.74 & & & \\
\hline & $3^{c l}$ & 2,348 & 26.52 & 4.76 & & & \\
\hline & $4^{d l}$ & 1,829 & 25.92 & 4.97 & & & \\
\hline \multirow[t]{4}{*}{ SAS } & $1^{\text {a) }}$ & 2,379 & 18.37 & 4.55 & 50.508 & 0.000 & $c>a, d>a, c>b, d>b$ \\
\hline & $2^{b)}$ & 2,235 & 18.22 & 4.19 & & & \\
\hline & $3^{c 1}$ & 2,357 & 19.31 & 3.99 & & & \\
\hline & $4^{d l}$ & 1,839 & 19.49 & 4.01 & & & \\
\hline \multirow[t]{4}{*}{ SPA } & $1^{\text {a) }}$ & 2,222 & 27.04 & 6.14 & 8.025 & 0.000 & $a>b, a>c, a>d$ \\
\hline & $2^{b)}$ & 2,093 & 26.20 & 5.76 & & & \\
\hline & $3^{c 1}$ & 2,326 & 26.52 & 6.07 & & & \\
\hline & $4^{d)}$ & 1,825 & 26.35 & 5.93 & & & \\
\hline \multirow[t]{4}{*}{ SSS } & $1^{\text {a) }}$ & 2,411 & 15.56 & 3.96 & 42.726 & 0.000 & $a>b, c>a, d>a, c>b, d>b$ \\
\hline & $2^{b)}$ & 2,234 & 15.16 & 3.70 & & & \\
\hline & $3^{c 1}$ & 2,345 & 16.16 & 3.50 & & & \\
\hline & $4^{d l}$ & 1,824 & 16.26 & 3.46 & & & \\
\hline \multirow[t]{4}{*}{ Total } & $1^{\text {al }}$ & 2,100 & 114.10 & 23.22 & 10.996 & 0.000 & $a>b, c>b, d>b$ \\
\hline & $2^{b l}$ & 2,002 & 111.80 & 21.07 & & & \\
\hline & $3^{c 1}$ & 2,218 & 115.56 & 20.84 & & & \\
\hline & $4^{d l}$ & 1,758 & 114.35 & 20.97 & & & \\
\hline
\end{tabular}

DREEM: Dundee Ready Education Environment Measure, SD: Standard deviation, SPL: Students' perceptions of learning, SPT: Students' perceptions of teachers, SAS: Students' academic self-perceptions, SPA: Students' perceptions of atmosphere, SSS: Students' social self-perceptions.

Table 7. Students' Perception of DREEM by Gender

\begin{tabular}{|c|c|c|c|c|c|c|}
\hline Factor & Gender & No. & Mean & SD & $t$ & $\mathrm{p}$-value \\
\hline \multirow[t]{2}{*}{ SPL } & Male & 5,189 & 26.16 & 6.70 & -0.419 & 0.675 \\
\hline & Female & 3,356 & 26.22 & 5.71 & & \\
\hline \multirow[t]{2}{*}{ SPT } & Male & 5,112 & 26.58 & 5.14 & -0.165 & 0.869 \\
\hline & Female & 3,310 & 26.60 & 4.71 & & \\
\hline \multirow[t]{2}{*}{ SAS } & Male & 5,211 & 18.94 & 4.45 & 3.668 & 0.000 \\
\hline & Female & 3,350 & 18.59 & 3.93 & & \\
\hline \multirow[t]{2}{*}{ SPA } & Male & 5,029 & 26.90 & 6.19 & 6.849 & 0.000 \\
\hline & Female & 3,196 & 25.97 & 5.62 & & \\
\hline \multirow[t]{2}{*}{ SSS } & Male & 5,205 & 15.94 & 3.80 & 5.644 & 0.000 \\
\hline & Female & 3,367 & 15.47 & 3.54 & & \\
\hline \multirow[t]{2}{*}{ Total } & Male & 4,808 & 114.59 & 22.65 & 3.146 & 0.002 \\
\hline & Female & 3,049 & 113.01 & 19.90 & & \\
\hline
\end{tabular}

DREEM: Dundee Ready Education Environment Measure, SD: Standard deviation, SPL: Students' perceptions of learning, SPT: Students' perceptions of teachers, SAS: Students' academic self-perceptions, SPA: Students' perceptions of atmosphere, SSS: Students' social self-perceptions. 
Table 8. Students' Perception of DREEM by Academic Achievement

\begin{tabular}{|c|c|c|c|c|c|c|c|}
\hline Factor & $\begin{array}{c}\text { Academic } \\
\text { achievement }\end{array}$ & No. & Mean & SD & $\mathrm{F}$ & $\mathrm{p}$-value & Scheffé \\
\hline \multirow[t]{3}{*}{ SPL } & High $^{\text {al }}$ & 3,838 & 26.89 & 6.31 & 59.547 & 0.000 & $b>c, a>c, a>b$ \\
\hline & Middle $^{\text {bl }}$ & 2,588 & 26.16 & 6.04 & & & \\
\hline & $\operatorname{Low}^{c \mid}$ & 2,038 & 25.01 & 6.63 & & & \\
\hline \multirow[t]{3}{*}{ SPT } & High $^{\text {a) }}$ & 3,779 & 26.76 & 5.01 & 5.172 & 0.000 & $a>c$ \\
\hline & Middle ${ }^{b /}$ & 2,559 & 26.56 & 4.85 & & & \\
\hline & Low $^{c l}$ & 2,010 & 26.32 & 5.14 & & & \\
\hline \multirow[t]{3}{*}{ SAS } & $\operatorname{High}^{\text {al }}$ & 3,841 & 20.00 & 4.05 & 344.703 & 0.000 & $b>c, a>c, a>b$ \\
\hline & Middle $^{\text {bl }}$ & 2,594 & 18.52 & 3.85 & & & \\
\hline & Low $^{c l}$ & 2,053 & 17.11 & 4.47 & & & \\
\hline \multirow[t]{3}{*}{ SPA } & High $^{\text {al }}$ & 3,691 & 27.11 & 5.95 & 43.278 & 0.000 & $b>c, a>c, a>b$ \\
\hline & Middle $^{\mathrm{bl}}$ & 2,500 & 26.56 & 5.74 & & & \\
\hline & Low $^{c l}$ & 1,975 & 25.50 & 6.30 & & & \\
\hline \multirow[t]{3}{*}{ SSS } & High $^{\text {al }}$ & 3,844 & 16.14 & 3.69 & 54.435 & 0.000 & $b>c, a>c, a>b$ \\
\hline & Middle $^{\mathrm{bl}}$ & 2,596 & 15.79 & 3.53 & & & \\
\hline & Low $^{c \mid}$ & 2,049 & 15.09 & 3.88 & & & \\
\hline \multirow[t]{3}{*}{ Total } & High $^{\text {al }}$ & 3,535 & 116.99 & 21.53 & 82.902 & 0.000 & $b>c, a>c, a>b$ \\
\hline & Middle ${ }^{b l}$ & 2,391 & 113.70 & 20.45 & & & \\
\hline & Low $^{\text {cl }}$ & 1,874 & 109.12 & 22.66 & & & \\
\hline
\end{tabular}

DREEM: Dundee Ready Education Environment Measure, SD: Standard deviation, SPL: Students' perceptions of learning, SPT: Students' perceptions of teachers, SAS: Students' academic self-perceptions, SPA: Students' perceptions of atmosphere, SSS: Students' social self-perceptions.

0.001)에서는 남학생이 여학생보다 높은 평균점수를 나타내 었다. 반면, $\mathrm{SPL}(\mathrm{t}=-0.419, \mathrm{p}>0.05)$ 과 $\mathrm{SPT}(\mathrm{t}=-0.165, \mathrm{p}>$ 0.05)는 남학생과 여학생 간의 유의미한 차이가 없었다(Table 7).

\section{6. 학업성적 수준에 따른 DREEM의 차이}

학생이 자신의 학업성적 수준을 상, 중, 하 3 단계로 구분한 후, 학업성적 수준에 따른 DREEM의 차이를 분석한 결과, $\mathrm{DREEM}$ 전체 평균점수는 상위권이 116.99점, 중위권이 113.70점, 하위권이 109.12점으로 나타나, 상위권 학생일수 록 점수가 높았다 $(\mathrm{F}=82.902, \mathrm{p}<0.001)$. 각 하위 요인별로 살 펴보면, $\mathrm{SPL}(\mathrm{F}=59.547, \mathrm{p}<0.001)$, SPT $(\mathrm{F}=5.172, \mathrm{p}<0.01)$, SAS $(F=344.703, p<0.001)$, SPA $(F=43.278, p<0.001)$, $\mathrm{SSS}(\mathrm{F}=54.435, \mathrm{p}<0.001)$ 에서도 모두 상위권 학생일수록 점 수가 높게 나타났다(Table 8). 즉, 상위권일수록 의학교육환 경을 더 긍정적으로 인식하는 것으로 나타났다.

\section{고찰}

본 연구에서는 우리나라 의대 및 의전원 전체 학생을 대상 으로 DREEM을 이용하여 의학교육환경에 대해 어떻게 인식 하고 있는지를 조사하여, 대학별, 하위 요인별, 문항별 분포를 살펴보고, 학제, 성별, 학년, 학업 성적 수준에 따라 어떠한 차 이가 있는지를 분석하였다. 그 결과를 요약하고 논의하면 다 음과 같다.

우리나라 학생들이 인식하는 DREEM의 전체 평균점수는 200점 만점에 약 114점(약 57\%)이다. 해석 기준으로 보면, 101 150점대인 긍정적인 영역에 해당되지만, 대학별 점수는 최고점수 137 점에서 최하점수 100 점까지의 분포를 보였고, 이 중 12개 대학(30\%)이 110점 미만이었고, 130점 이상인 대 학은 1 개 대학뿐이었다. 점수 기준으로는 긍정적인 편이라고 해석할 수 있겠지만, 대학 간 편차가 있음을 알 수 있다. 선행 연구에 의하면, 영국의 던디 의과대학[11] 등 유럽의 대학들 
은 130 점 이상의 점수를 나타내었다. 반면, 우리나라는 전반 적으로 스리랑카[9], 나이지리아[10], 인도[3,14] 등의 나라와 비슷한 점수 분포이다. 여러 선행 연구를 종합해 볼 때, 20세 기 이후 의학교육의 변화 방향에 맞추어 오랫동안 학습자 중 심 교육, 통합교육, 문제바탕학습 등의 교육과정을 운영해 온 대학들이 더 높은 점수를 보였는데, 우리나라도 이러한 방향 으로 의학교육과정을 개편하려는 노력을 해 왔고 그 동안 많 은 변화가 있었다[12]. 하지만, 학생들의 의학교육환경에 대 한 인식은 그에 미치지 못한다는 측면에서, 지난 세기 동안 변 화와 발전을 거듭해 온 우리나라 의학교육에 대한 현재의 모 습과 나아갈 방향에 대한 깊이 있는 성찰을 해 볼 수 있는 계 기를 제공해 준다고 할 수 있겠다.

이러한 관점에서 각 문항별로 DREEM 해석 기준에서 평균 점수가 2점 이하로 낮게 나온 10 문항을 가지고 고찰하고자 한 다. 하위 요인 중 SPL이 2점 이하인 문항이 가장 많았다. 학 생들은 장기기억보다는 단기기억의 학습이 강조된다고 인식 하며(문항 47), 수업이 학생 중심적이지 않고(문항 13), 교수 중심적이며(문항 48), 수업이 단순암기학습을 지나치게 강조 한다(문항 25)고 인식하였다. 이는 의대교육이 여전히 교수 중심적이며 단순 암기를 강조하는 환경에 놓여 있음을 보여 주는 것이다. 선행 연구에서도 전통적인 교육과정을 운영하 는 대학이 주로 이러한 문항에서 문제를 나타내었다[8,12]. 그 동안 우리나라 대부분의 의과대학은 학습자 중심의 교육 과정을 강조하였고, 문제바탕학습이나 팀바탕학습과 같은 다 양한 교육방법이 확산되고, 이에 맞는 교육환경을 구축하였 다[12]. 즉, 의학교육이 추구하는 변화 방향과는 다르게 학생 들은 학습과 관련하여 여전히 전통적인 교육과정을 운영하는 대학이 지닌 문제들을 그대로 인식하고 있었다. 이는 의학교 육이 질적 변화와 내실화에 더 많은 노력을 기울여야 한다는 측면에서 시사하는 바가 크다.

학생들은 의과대학 교수들에 대해 교수들이 충분한 지식을 가지고 있다(문항 2)에 가장 높은 점수를 주었지만, 반면 권위 주의적이라고 인식하고 있다(문항 9). 교수의 권위주의적인 측면은 교육환경에서 가장 변화가 어려운 부분 중 하나라고 지적한 바 있다 $[3,14,15]$. 대학은 교수가 과거 교육경험에 의 존하여 가르치며 권위를 내세우기보다, 교육과정이나 의학교 육환경의 변화에 요구되는 적절한 교수법을 개발하고, 학생
과 소통할 수 있도록 지속적으로 다양한 교수개발프로그램을 제공하여야 할 것이다. 또한 주목할 것은 의과대학생활에서 스트레스를 받고 있지만(42번), 스트레스를 받고 있는 학생에 대한 지원체계는 부족하다(문항 3)에 가장 낮은 점수를 주었 다. 대학은 교수개발을 위한 노력과 더불어 학생의 학습과 복 지에 대해 관심을 갖고 지원 체계를 구축하는 데 많은 노력이 필요하다.

학생들의 개인 특성에 따라 DREEM 점수에서 차이가 있었 다. 의전원생이 의대생에 비해 DREEM 전체 평균점수와 하 위 요인에 대해 더 높은 점수를 나타내었다. 한 연구에 의하 면, 고등학교 졸업 후 바로 대학을 입학한 학생과 그렇지 않은 학생을 비교한 결과, 후자의 학생들이 더 긍정적으로 인식하 는 것으로 나타나 본 연구 결과를 지지해 준다[16]. 마찬가지 로 의전원생은 연령이 높고, 성숙하며, 입학 이전에 다양한 교 육 경험이 있고, 도전적이며, 높은 학업동기를 가지고 있기 때 문에[17], 이러한 적극적이고 긍정적인 태도로 의학교육환경 에 대해서도 좀 더 수용적이고 긍정적으로 인식하였을 것으 로 판단된다.

학년에 따라 DREEM 전체 평균점수와 하위 요인 점수에 차이가 있었다. SPT와 SPA에 대해서는 1학년 때가 가장 높 고 점차적으로 낮아지는 경향이 있는데, 이는 여러 연구에서 도 마지막 학년이 가장 낮은 점수를 나타낸 결과와 일치한다 [14]. SAS와 SSS는 학년이 올라갈수록 높아지는 것을 알 수 있다. 학년이 올라갈수록 학습 결과를 통해 학업에 대한 자신 감이 더 높아지며, 학교 생활에 대한 적응도 높아진다고 볼 수 있다. DREEM 전체 평균점수에서는 2 학년이 가장 낮은 점수 를 나타내었다. 대체적으로 교육과정에서 2학년은 주로 임상 실습 전 시기로 의학교육환경을 가장 긍정적으로 인식한다는 연구 결과와는 차이를 보인다[18]. 우리나라의 경우, DREEM 전체 평균 점수뿐만 아니라 하위 요인인 학습(SPL)에서도 가 장 낮은 점수를 보여, 2 학년 시기의 의학교육환경에 대한 문 제점과 원인을 찾아볼 필요가 있다.

남학생이 여학생보다 DREEM 전체 평균점수와 하위 요인 인 SAS, SPA, SSS에서 더 높은 점수를 나타내었다. 던디대 학과 중동지역의 3개 대학을 비교한 연구에서[11], 전통적인 교육과정을 운영하는 중동의 3 개 대학은 남학생이 여학생보 다 높은 인식도를 보였다는 연구 결과와 일치한다. 반면, 같은 
연구에서 비교집단이었던 학습자 중심의 교육과정 운영을 대 표하는 던디 대학은 남녀 차이가 없었다. 본 연구 결과는 여러 연구에서 여학생들이 교육환경을 훨씬 더 친근하고 긍정적으 로 인식한다는 연구와는 상반된 결과이다[11,16]. 또한 몇몇 연구에서는 성별에 따른 DREEM에 유의한 차이가 없다는 결 과도 보고된 바 있다[11,15,19]. 이처럼 의학교육환경 인식에 있어서 성별 차이는 일치된 결론을 내리기가 어렵지만, 교육 환경에 대해 성별에 관계없이 긍정적인 인식을 할 수 있도록 개선하는 것이 바람직할 것이다.

학업성적 수준을 3 단계로 구분하였을 때, 학업성적 수준이 높은 집단이 DREEM 전체 평균점수도 높았다. 즉, 학업성적 이 상위권인 학생들이 의학교육환경에 대해서도 긍정적으로 인식한다는 연구 결과를 지지해 준다[3]. 학업성취가 높은 학 생들은 횔씬 자기주도적이기 때문에 학업성취가 낮은 학생들 보다는 성인 학습자의 수준에 있다고 할 수 있고 의학교육환 경을 보다 학생 중심적으로 인식하는 경향이 있다. 반면, 성취 도가 낮은 학생들은 교육환경에 만족하지 못하는 특성이 있 다[15]. 이처럼 동일한 환경에 대해서 성취수준에 따라 환경 인식에 분명한 차이가 있기 때문에, 성취도가 낮은 학생에 대 한 교육적 배려가 필요할 것이다.

이처럼 본 연구는 우리나라 전체 의대생들의 의학교육환경 에 대한 학생들의 인식 정도를 파악하고, 대학 간 상대 비교뿐 만 아니라 국제 비교를 위한 기초자료를 제공함으로써 의학 교육환경의 중요성을 인식시키고 개선 방안을 마련하는 데 활용할 수 있도록 하였다는 측면에서 의의가 있다. 그럼에도 불구하고 본 연구에서 학업성적 수준은 의학교육환경 인식에 중요한 변인임에도 불구하고, 실제 성적이 아닌 학생들의 응 답에 기준하여 조사하였기 때문에 한계가 있다. 또한 번안한 문항이기 때문에 전문가의 충분한 검토 과정을 거쳤다 하더 라도 의미상 불확실하고 모호한 측면을 완전히 배제할 수 없 다는 제한점이 있다. 교육환경에 대해 학생들이 기대하는 인 식도와 실제 인식도에 차이가 있고, 실제 인식도가 더 낮은 편 이기 때문에[19], 주기적인 조사를 통해 그 차이를 개선하려 는 노력이 필요할 것이다. 또한 의학교육환경은 의대생뿐만 아니라 전공의 수련이나 의사국가시험과 관련이 있지만[20], 국내에서는 아직 연구가 부족한 실정이다. 따라서 향후에는 의대생뿐만 아니라 다양한 보건의료 관련 전공자를 대상으로
확대하고, 의학교육환경과 관련이 있는 다양한 요인들을 종 합적으로 고려한 추가 연구가 필요하다. 마지막으로 DREEM 이 문화 보편적인 검사이지만, 우리나라에 맞게 타당화하는 추가 연구가 필요할 것이다.

Acknowledgements: None.

Funding: This research was supported by the Korean Society of Medical Education, Korean Association of Medical Colleges, and Korean Academy of Medical Sciences.

Conflicts of interest: None.

\section{REFERENCES}

1. Hutchinson L. Educational environment. BMJ 2003; 326: $810-812$.

2. Genn JM. AMEE medical education guide No. 23 (part 1): curriculum, environment, climate, quality and change in medical education: a unifying perspective. Med Teach 2001; 23: 337-344.

3. Mayya S, Roff S. Students' perceptions of educational environment: a comparison of academic achievers and under-achievers at Kasturba Medical College, India. Educ Health (Abingdon) 2004; 17: 280-291.

4. Roff S, McAleer S. What is educational climate? Med Teach 2001; 23: 333-334.

5. Roff S, McAleer S, Harden RM, Al-Qahtani M, Ahmed AU, Deza H, Groenen G, Primparyon P. Development and validation of the Dundee Ready Education Environment Measure (DREEM). Med Teach 1997; 19: 295299.

6. Roff S. The Dundee Ready Educational Environment Measure (DREEM): a generic instrument for measuring students' perceptions of undergraduate health professions curricula. Med Teach 2005; 27: 322-325. 
7. Miles S, Swift L, Leinster SJ. The Dundee Ready Education Environment Measure (DREEM): a review of its adoption and use. Med Teach 2012; 34: e620-e634.

8. Zawawi AH, Elzubeir M. Using DREEM to compare graduating students' perceptions of learning environments at medical schools adopting contrasting educational strategies. Med Teach 2012; 34 Suppl 1: S25-S31.

9. Jiffry MT, McAleer S, Fernando S, Marasinghe RB. Using the DREEM questionnaire to gather baseline information on an evolving medical school in Sri Lanka. Med Teach 2005; 27: 348-352.

10. Roff S, McAleer S, Ifere OS, Bhattacharya S. A global diagnostic tool for measuring educational environment: comparing Nigeria and Nepal. Med Teach 2001; 23: 378-382.

11. Al-Hazimi A, Zaini R, Al-Hyiani A, Hassan N, Gunaid A, Ponnamperuma G, Karunathilake I, Roff S, McAleer S, Davis M. Educational environment in traditional and innovative medical schools: a study in four undergraduate medical schools. Educ Health (Abingdon) 2004; 17: 192-203.

12. Yang EB, Meng KH. Five suggestions for future medical education in Korea. Korean J Med Educ 2014; 26: 167-178.

13. McAleer S, Roff S. A practical guide to using the Dundee Ready Education Environment Measure (DREEM). In: Genn JM, ed. Curriculum, environment, climate, quality and change in medical education: a unifying perspective. Dundee, Scotland: Association for Medical Education in
Europe; 2001. p 29-33.

14. Kohli V, Dhaliwal U. Medical students' perception of the educational environment in a medical college in India: a cross-sectional study using the Dundee Ready Education Environment questionnaire. J Educ Eval Health Prof 2013; 10: 5 .

15. Dashputra A, Chari S, Gade S. Perception of educational environment in a private medical college in central India. Int J Edu Sci 2014; 6: 489-496.

16. Brown T, Williams B, Lynch M. The Australian DREEM: evaluating student perceptions of academic learning environments within eight health science courses. Int J Med Educ 2011; 2: 94-101.

17. McCrorie P. Graduate students are more challenging, demanding, and questioning. BMJ 2002; 325: 676.

18. Demirören M, Palaoglu O, Kemahli S, Ozyurda F, Ayhan IH. Perceptions of students in different phases of medical education of educational environment: Ankara University Faculty of Medicine. Med Educ Online 2008; 13: 8.

19. Miles S, Leinster SJ. Medical students' perceptions of their educational environment: expected versus actual perceptions. Med Educ 2007; 41: 265-272.

20. Tokuda Y, Goto E, Otaki J, Jacobs J, Omata F, Obara H, Shapiro M, Soejima K, Ishida Y, Ohde S, Takahashi O, Fukui T. Undergraduate educational environment, perceived preparedness for postgraduate clinical training, and pass rate on the National Medical Licensure Examination in Japan. BMC Med Educ 2010; 10: 35. 
Appendix 1. Dundee Ready Education Environment Measure

\begin{tabular}{|c|c|c|c|c|c|}
\hline 문 항 & $\begin{array}{c}\text { 매우 } \\
\text { 그렇다 }\end{array}$ & 그렇다 & 보통 & 아니다 & $\begin{array}{c}\text { 전혀 } \\
\text { 아니다 }\end{array}$ \\
\hline 1. 나는 수업에 참여하도록 격려 받고 있다. & 4 & 3 & 2 & 1 & 0 \\
\hline 2. 교수님들은 풍부한 지식을 갖고 있다. & 4 & 3 & 2 & 1 & 0 \\
\hline 3. 스트레스 받는 학생을 위한 지원 체계가 잘 갖춰져 있다. & 4 & 3 & 2 & 1 & 0 \\
\hline 4. 나는 너무 힘들어서 수업을 즐길 수가 없다. & 4 & 3 & 2 & 1 & 0 \\
\hline 5. 과거에 내가 공부할 때 사용했던 학습 전략방법이 지금 의대 공부를 하는 데도 효과적이다. & 4 & 3 & 2 & 1 & 0 \\
\hline 6. 교수님들은 환자를 관대하게 대하시는 편이다. & 4 & 3 & 2 & 1 & 0 \\
\hline 7. 수업은 학습에 대한 흥미를 불러일으켜주는 편이다. & 4 & 3 & 2 & 1 & 0 \\
\hline 8. 교수님들은 학생을 무시하는 편이다. & 4 & 3 & 2 & 1 & 0 \\
\hline 9. 교수님들은 권위주의적인 편이다. & 4 & 3 & 2 & 1 & 0 \\
\hline 10. 나는 올해 진급할 수 있으리라고 확신한다. & 4 & 3 & 2 & 1 & 0 \\
\hline 11. 병동 실습을 할 때 분위기가 편안하다. & 4 & 3 & 2 & 1 & 0 \\
\hline 12. 우리 학교의 학년별 학기별 시간표(학사일정과 교육과정)는 잘 짜여져 있다. & 4 & 3 & 2 & 1 & 0 \\
\hline 13. 수업은 학생 중심적이다. & 4 & 3 & 2 & 1 & 0 \\
\hline 14. 나는 이번 학기가 별로 지루하지 않다. & 4 & 3 & 2 & 1 & 0 \\
\hline 15. 나는 학교에 친한 친구들이 있다. & 4 & 3 & 2 & 1 & 0 \\
\hline 16. 수업은 나의 역량을 개발시키는 데 도움이 된다. & 4 & 3 & 2 & 1 & 0 \\
\hline 17. 우리 학교에서는 시험 때 커닝이 문제가 된다. & 4 & 3 & 2 & 1 & 0 \\
\hline 18. 교수님들은 환자와 의사소통을 잘 하는 편이다. & 4 & 3 & 2 & 1 & 0 \\
\hline 19. 나의 의대/의전원에서의 생활(동료 관계)은 원만하다. & 4 & 3 & 2 & 1 & 0 \\
\hline 20. 수업의 초점이 명확하다. & 4 & 3 & 2 & 1 & 0 \\
\hline 21. 나는 의사로서의 전문성을 갖추기 위해 잘 준비되고 있다고 생각한다. & 4 & 3 & 2 & 1 & 0 \\
\hline 22. 수업은 나의 자신감을 증진시키는 데 도움이 된다. & 4 & 3 & 2 & 1 & 0 \\
\hline 23. 수업시간의 분위기는 편안하다. & 4 & 3 & 2 & 1 & 0 \\
\hline 24. 수업시간은 유용하게 잘 짜여졔구성되에 있다. & 4 & 3 & 2 & 1 & 0 \\
\hline 25. 수업은 사실 자체의 단순암기학습(단순 지식 전달)을 지나치게 강조한다. & 4 & 3 & 2 & 1 & 0 \\
\hline 26. 지난 해 공부한 내용이 올해 공부를 하는 데 유용하였다. & 4 & 3 & 2 & 1 & 0 \\
\hline 27. 나는 내가 필요로 하는 모든 것을 기억할 수 있다. & 4 & 3 & 2 & 1 & 0 \\
\hline 28. 나는 좀처럼 외로움을 느끼지 않는다. & 4 & 3 & 2 & 1 & 0 \\
\hline 29. 교수님은 학생들에게 피드백을 잘 해 주신다. & 4 & 3 & 2 & 1 & 0 \\
\hline 30. 의대/의전원 생활 중에 대인관계 기술을 향상시킬 기회가 있다. & 4 & 3 & 2 & 1 & 0 \\
\hline 31. 나는 의사로서의 전문성에 있어 공감할 수 있는 많은 것을 배우고 있다. & 4 & 3 & 2 & 1 & 0 \\
\hline 32. 교수님들은 건설적인 조언을 해 주시는 편이다. & 4 & 3 & 2 & 1 & 0 \\
\hline 33. 나는 교실 내에서 허물없이 편안함을 느낀다. & 4 & 3 & 2 & 1 & 0 \\
\hline 34. 세미나나 소그룹 수업의 분위기는 편안하다. & 4 & 3 & 2 & 1 & 0 \\
\hline 35. 나는 의대/의전원에서 실망스러운 경험을 하곤 한다. & 4 & 3 & 2 & 1 & 0 \\
\hline 36. 의대/의전원 분위기가 내가 잘 집중할 수 있는 분위기이다. & 4 & 3 & 2 & 1 & 0 \\
\hline 37. 교수님은 분명한 예시를 보여주신다. & 4 & 3 & 2 & 1 & 0 \\
\hline 38. 나는 수업의 학습목표를 명확하게 이해하고 있다. & 4 & 3 & 2 & 1 & 0 \\
\hline 39. 교수님들은 수업 중에 화를 내기도 한다. & 4 & 3 & 2 & 1 & 0 \\
\hline 40. 교수님은 수업준비를 잘 하시고 수업에 들어오신다. & 4 & 3 & 2 & 1 & 0 \\
\hline 41. 의대/의전원에서 나의 문제해결능력은 향상되고 있다 & 4 & 3 & 2 & 1 & 0 \\
\hline 42. 나는 학업에 대해 스트레스를 받기보다는 즐기는 편이다. & 4 & 3 & 2 & 1 & 0 \\
\hline 43. 학교/교실의 분위기는 내가 학습하고자 하는 동기를 불러일으켜 준다. & 4 & 3 & 2 & 1 & 0 \\
\hline
\end{tabular}


Kwi Hwa Park, et al: Perception of educational environment in medical school

\begin{tabular}{|c|c|c|c|c|c|}
\hline 문 항 & $\begin{array}{c}\text { 매우 } \\
\text { 그렇다 }\end{array}$ & 그렇다 & 보통 & 아니다 & $\begin{array}{c}\text { 전혀 } \\
\text { 아니다 }\end{array}$ \\
\hline 44. 수업은 내가 능동적인 학습자가 되도록 북돋아준다. & 4 & 3 & 2 & 1 & 0 \\
\hline 45. 내가 학습해 온 많은 내용들은 앞으로 의사직을 수행하는 것과 관련이 있는 것 같다. & 4 & 3 & 2 & 1 & 0 \\
\hline 46. 나의 학교 편의시설(기숙사 등)은 쾌적하고 좋다. & 4 & 3 & 2 & 1 & 0 \\
\hline 47. 장기기억의 학습이 단기기억의 학습보다 강조된다. & 4 & 3 & 2 & 1 & 0 \\
\hline 48. 수업이 너무 교수 중심적이다. & 4 & 3 & 2 & 1 & 0 \\
\hline 49. 내가 원한다면 언제든지 질문을 할 수 있는 분위기다. & 4 & 3 & 2 & 1 & 0 \\
\hline 50. 학생들은 교수님들을 힘들게 하는 편이다. & 4 & 3 & 2 & 1 & 0 \\
\hline
\end{tabular}

